\title{
Herramienta para la elaboración del TFG en un entorno estadístico mediante Moodle ("book")
}

\author{
Tool for the elaboration of the TFG in a statistical environment through \\ Moodle ("book")
}

\section{Susana Marín Feria, Salvador Torra Porras, Francisco Llorente Galera, Elena Rico Gómez, Margarita Carrillo López}

\author{
Departamento Econometría, Estadística y Economía Aplicada \\ Universidad de Barcelona \\ Diagonal, 690, 08034, Barcelona \\ smarin@ub.edu storra@ub.edu fllorente@ub.edu \\ erico@ub.edu mcarrillo@ub.edu
}

\begin{abstract}
Resumen
El objetivo del artículo es presentar un trabajo de innovación docente, realizado por un grupo de profesores del Departamento de Econometría, Estadística y Economía Aplicada de la Facultad de Economía y Empresa de la UB (DOI: 10.1344/401.000002955), consistente en la producción de un libro de Moodle para dar soporte a aquellos estudiantes de A.D.E. (Administración y Dirección de Empresas) que se enfrentan, al final de sus estudios, al reto de realizar su TFG (Trabajo de Fin de Grado). Está especialmente diseñado para aquellos que tienen pensado realizar un TFG en el que las técnicas estadísticas vayan a tener un papel relevante en el contenido de su estudio.

Efectivamente, constatada la falta de conocimientos que manifiestan muchos de estos estudiantes sobre el proceso a seguir a la hora de elaborar un trabajo de investigación de una cierta entidad, como lo es el TFG, y la ansiedad que esa situación les genera, se decidió producir este recurso docente, accesible sin coste desde la plataforma Moodle con la que los estudiantes ya están familiarizados y que, además, tiene la ventaja añadida de disponer de una serie de herramientas auxiliares interesantes que pueden potenciar aún más la utilidad del material.

El libro viene a ser un manual, que se pretendió conciso y práctico, para resolver airosamente la cuestión del TFG de principio a fin: desde la producción de la idea clave del trabajo (desde la definición del tema), pasando por la generación/recogida de datos y su posterior análisis, y por la imprescindible extracción de conclusiones, hasta la etapa final de redacción de la memoria y presentación pública del trabajo que implica también la confección de un poster.

De forma paralela al libro se elaboraron una serie de cuestionarios para medir: i) los conocimientos previos de los estudiantes sobre el tema, ii) los conocimientos adquiridos tras el uso del material y iii) la valoración por parte de los estudiantes del recurso. Sin embargo, todavía no es posible evaluar en qué medida la herramienta ha sido útil a aquellos para la que se creó: el período docente 2017-2018, es el primer curso académico en que se pone el material a disposición del alumnado. A final de curso será posible realizar ya un primer análisis de los datos proporcionados por esos cuestionarios.
\end{abstract}

Palabras clave: TFG, ADE, Técnicas de análisis estadístico, Libro de Moodle.

\begin{abstract}
The objective of the article is to present a teaching innovation work, carried out by a group of professors from the Department of Econometrics, Statistics and Applied Economics of the Faculty of Economics and Business of the UB (DOI: 10.1344/401.000002955), consisting of the production of a Moodle book to support those ADE students (Business Administration and Management) who face, at the end of their studies, the challenge of completing their TFG (Final Degree Project). It is specially designed for those who plan to perform a TFG in which statistical techniques will have a relevant role in the content of their study.
\end{abstract}


Indeed, the lack of knowledge manifested by many of these students about the process to follow when developing a research work of a certain entity, such as the TFG, and the anxiety that this situation generates, was decided upon. this teaching resource, accessible without cost from the Moodle platform with which the students are already familiar and which, in addition, has the added advantage of having a series of interesting auxiliary tools that can further enhance the usefulness of the material.

The book becomes a manual, which was intended to be concise and practical, to resolve the issue of the TFG from beginning to end: from the production of the key idea of the work (from the definition of the theme), through the generation / collection of data and its subsequent analysis, and by the essential extraction of conclusions, until the final stage of writing the report and public presentation of the work that also involves the making of a poster.

Parallel to the book, a series of questionnaires were prepared to measure: i) the students 'prior knowledge on the subject, ii) the knowledge acquired after the use of the material and iii) the students' assessment of the resource. However, it is still not possible to evaluate to what extent the tool has been useful to those for whom it was created: the teaching period 2017-2018 is the first academic year in which the material is made available to students. At the end of the course it will be possible to carry out a first analysis of the data provided by these questionnaires.

Key words: TFG, ADE, Statistical Analysis Techniques, Moodle Book.

\section{Introducción}

Como es sabido, los estudiantes universitarios para finalizar sus estudios de Grado han de realizar un trabajo de una cierta dimensión, que permita demostrar que disponen de unos conocimientos y unas habilidades (o competencias) suficientes para el ejercicio de la profesión. Este trabajo de fin de grado (TFG) puede ser de diferente tipo pero habitualmente es un trabajo de investigación en el sentido clásico del término ${ }^{1}$.

En el caso de los estudios de ADE de la UB, a los estudiantes se les asigna un tutor, en función de la temática particular que hayan escogido para este TFG y a lo largo de un semestre (cuatrimestre) tienen la posibilidad de realizar el trabajo contando con el soporte y la orientación de este profesor, en el marco de una especie de evaluación continuada de esta asignatura especial que es el TFG ${ }^{2}$. Así, compartirán sesiones de trabajo y experiencias con aquellos otros estudiantes (compañeros) que hayan optado por la misma temática de TFG.

En este contexto, la experiencia como profesores-tutores de TFG de algunos de nosotros nos ha permitido conocer de primera mano las importantes limitaciones con las que muchos de estos estudiantes afrontan el reto de la realización de su TFG. Estas limitaciones suponen serias dificultades para mostrar un progreso adecuado en esta asignatura especial de TFG y, también, son una fuente importante de ansiedad para ellos. Hay que decir que estos estudiantes de ADE no cuentan, en su Plan de Estudios, con una asignatura específica sobre metodología de investigación en la empresa (o en las CCSS) y que la mayoría no ha realizado más de 2 o 3 trabajos de cierta entidad a lo largo de los estudios que están a punto de finalizar. Por otro lado, a algunos estudiantes adolecen de no tener frescos algunos conocimientos básicos que aprendieron en su momento en el marco de las asignaturas de Estadística impartidas dentro de su ciclo formativo del Grado.

Dar respuesta a esta situación insatisfactoria, fue la razón por la que los autores de este artículo decidimos crear, en el marco de un proyecto de innovación docente de la universidad, que fue aceptado como tal, la

\footnotetext{
${ }^{1}$ Otras opciones son que se trate de una revisión de la bibliografía sobre un tema, que sea un estudio de mercado o que consista en una memoria de prácticas, por citar algunas alternativas.

${ }^{2}$ Existe la posibilidad de renuncia a esta opción por parte del estudiante, de realizar el TFG por cuenta propia, sin ayuda del tutor, pero lo cierto es que es una opción muy residual entre los estudiantes, pensada especialmente para aquellos que trabajan o realizan prácticas empresariales.
} 
herramienta que aquí presentamos: un libro en la plataforma Moodle para asistir a los estudiantes de ADEUB que han de realizar su TFG en la temática de Técnicas estadísticas en el ámbito empresarial. El libro (manual) pretende explicar las diferentes fases de la realización de un trabajo de investigación, de principio (decisión del tema de investigación) a fin (redacción de la memoria) teniendo un peso importante, las técnicas de análisis estadístico; haciéndolo de forma clara y concisa, para que pueda utilizarlo en el espacio de tiempo limitado del semestre de matriculación del TFG; está adaptado a los estudiantes (de Empresa) a los que va dirigido y, por último, está disponible online, en la plataforma Moodle con la que los estudiantes ya están muy familiarizados, integrado en el campus virtual de la asignatura. Y además, para permitir una evaluación del impacto y de la aceptación de la herramienta (y cumplir con uno de los requisitos básicos del programa de innovación), junto al libro se diseñan una serie de cuestionarios que se pasarán a los estudiantes a principio y a final de curso.

El proyecto finalizó el curso académico 2016-2017 y es en este nuevo curso (17-18; segundo semestre) que se presentará a un primer grupo de estudiantes, lo cual no nos permite todavía evaluar el comentado impacto del recurso ni conocer la valoración de su utilidad por parte de los estudiantes, pero estamos convencidos de que los resultados serán positivos. Los primeros datos al respecto los tendremos a la finalización del presente curso.

En un primer apartado (después de esta introducción) se especifica el contexto en el que se enmarca este proyecto de los TFG de los estudiantes del Grado de ADE de la Universidad de Barcelona y la herramienta elaborada. Posteriormente se detallan las características que deben presentar estos trabajos y cómo está previsto que se desarrolle a lo largo del semestre la tutorización y la evaluación continuada de los mismos. Seguidamente, describimos con detalle el proyecto llevado a cabo (el libro y los cuestionarios que lo complementan). Para acabar, se destacan los puntos fuertes del mismo y, a la vez, posibles mejoras a realizar en un futuro. Cierran el artículo unas conclusiones, la bibliográfica y unos anexos que muestran las características de los materiales producidos en este proyecto.

\section{Contexto: el TFG en los estudios del Grado de ADE (UB)}

\section{Motivación y competencias del TFG y tipología de trabajos}

Como exige la normativa ${ }^{3}$, los estudiantes del Grado de ADE de la UB han de realizar necesariamente un trabajo de fin de grado (TFG) para completar los estudios y poder obtener la titulación. Sobre las características de este trabajo se indica lo siguiente: "EI TFG ha de ser una herramienta para poder evaluar si el estudiante ha integrado los conocimientos propios de la titulación y si ha adquirido, también, todas aquellas competencias que se le asocian (al título), las competencias específicas, como aquellas otras más transversales y generales. EI TFG es, también, una última instancia (oportunidad) de formación para el estudiante en el marco de los estudios de grado que está a punto de finalizar".

Las competencias transversales que se asocian al título de ADE son las siguientes:

- Capacidad de aprendizaje y de responsabilidad: capacidad de análisis, de síntesis, de visión global, de aplicación de los conocimientos adquiridos a la práctica, capacidad de tomar decisiones, capacidad de adaptación a nuevas situaciones.

- Capacidad creativa y emprendedora: capacidad de formular, de diseñar y de gestionar proyectos; capacidad de buscar e integrar nuevos conocimientos y actitudes.

- Capacidad comunicativa: capacidad de expresarse oralmente y por escrito, dominado el lenguaje especializado; capacidad de buscar, usar e integrar la información.

\footnotetext{
${ }^{3}$ Normativa de Treball de Final de Grau de la Facultat d'Economia i Empresa de la Universitat de Barcelona.
} 
- Capacidad de utilización de las nuevas tecnologías de la información y la comunicación (TIC) en el ámbito profesional.

- Capacidad para llevar una buena administración del tiempo.

- Compromiso ético.

No se exige que el TFG sea un trabajo de investigación de altísimo nivel (de hecho, puede no ser un trabajo de investigación en el sentido clásico del término), de gran originalidad (inédito) y relevancia. Esas deberían ser las características de un trabajo de postgrado o, quizás, de uno de doctorado (de una tesis). Al TFG no se le exige, obviamente, tanto.

La normativa contempla que el TFG de los estudios de ADE (UB) puede presentar alguno de los siguientes formatos:

- El de trabajo de investigación (en el sentido clásico del término).

- El de una revisión de la literatura sobre un tema.

- El de una simulación.

- El de un plan de empresa (Business plan)

- El de un caso de estudio (case study).

- El de una memoria vinculada a unas prácticas externas (en una empresa) curriculares.

El recurso docente elaborado (el libro de Moodle) fue diseñado para aquellos TFG del primer tipo mencionado: aquellos que representan un trabajo de investigación típico que, por otro lado, es la opción escogida de forma mayoritaria por el alumnado. Como se indica en el apartado final, no descartamos, si el recurso es valorado positivamente por el alumnado, repetir el ejercicio para algún otro tipo de TFG.

\section{Organización y desarrollo del TFG}

La organización académica del TFG se compara a la de una asignatura; una asignatura especial, no obstante. Así, el TFG cuenta con un Plan Docente, a cada estudiante se le asigna un tutor (en función de la temática particular que haya escogido) y es posible seguir una evaluación continuada (en contraposición a la posibilidad de realizar el trabajo por libre y tener una evaluación única). La evaluación continuada exige mantener a lo largo del semestre una serie de entrevistas o sesiones de trabajo con el tutor, otra serie de sesiones en grupo con otros estudiantes que han optado por la misma temática e ir presentando diferentes materiales de trabajo que muestren el avance en la investigación y que el tutor utilizará para evaluar el progreso y decidir, finalmente, la nota final (de evaluación continuada) del estudiante. La Figura 1 muestra la planificación de esta evaluación continuada del $\mathrm{TFG}^{4}$.

Las tareas del tutor son las siguientes:

- Actuar como dinamizador y facilitador de todo el proceso (de realización del TFG)

- Ayudar al estudiante a escoger y delimitar bien el tema y los objetivos del trabajo. (Si se considera conveniente proponer, incluso, un cambio de temática/grupo)

- Ayudar al estudiante a planificar el trabajo mediante un calendario.

- Orientar al estudiante en cuanto a la metodología a seguir y los métodos a aplicar.

- Ayudar al estudiante en la obtención de las conclusiones.

- Realizar el seguimiento de la evolución del trabajo.

- Elaborar un dosier con todo el material que le vaya presentado el estudiante.

- Evaluar la evolución del trabajo completando los correspondientes informes parciales normalizados.

\footnotetext{
${ }^{4}$ En el cuadro de la Figura 1 el acrónimo PPE significa Propuesta de Proyecto de Estudio.
} 
- Entregar un informe final, también normalizado, con la valoración global de todo el trabajo realizado por el estudiante.

- Participar en la valoración final (calificación) del TFG, de acuerdo a lo establecido por la normativa.

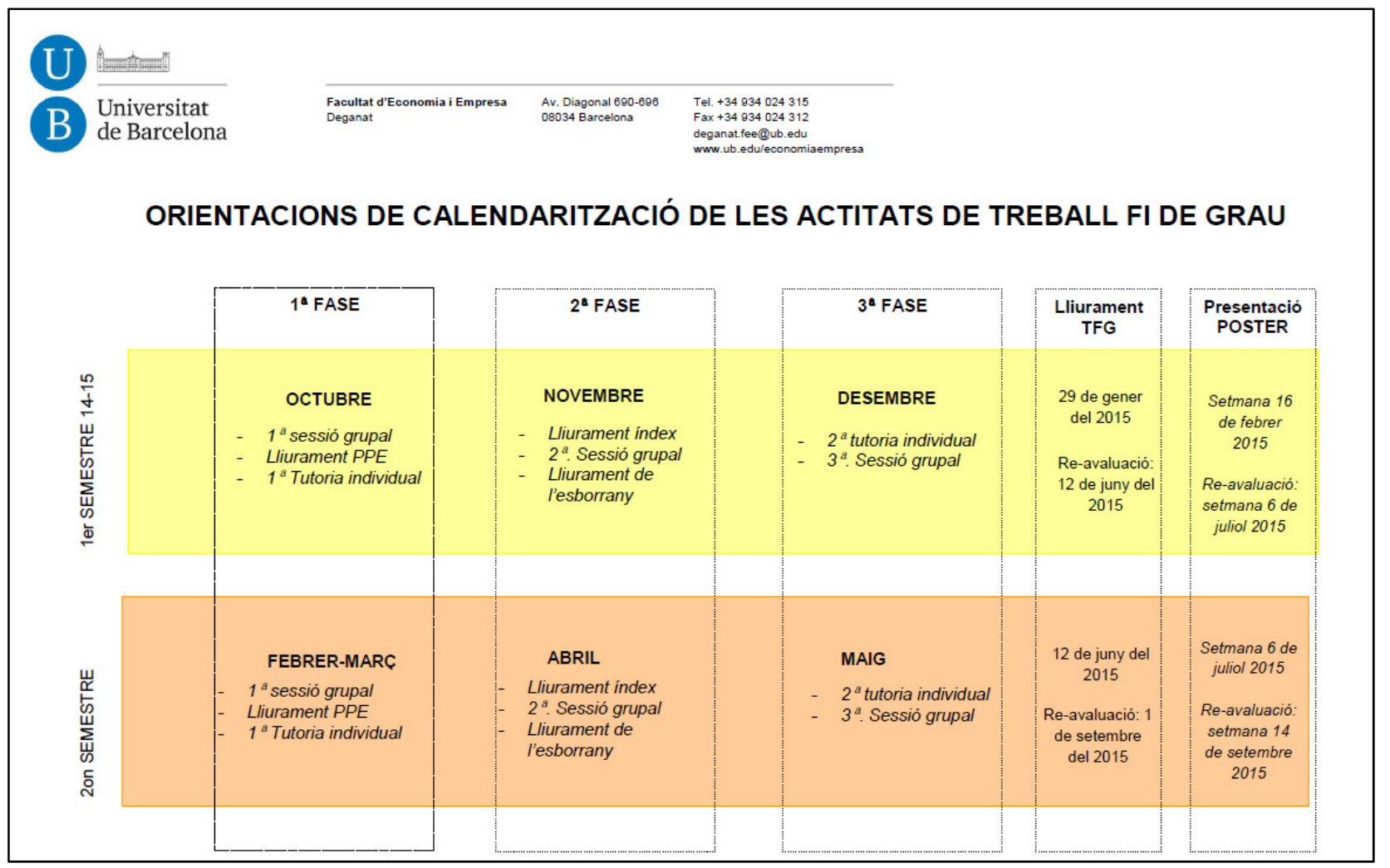

Figura 1: Planificación de la evaluación continuada del TFG

Añadir que existen dos modalidades de evaluación en función de que el trabajo (TFG) se presente o no públicamente. Si no se presenta en público el trabajo la nota no puede superar el 6,9 (el tutor podría dar, de hecho, una nota superior al trabajo pero la nota máxima en el expediente no podría superar ese 6,9). Para obtener una nota superior el estudiante tiene que presentar públicamente, ante un tribunal, el trabajo (lo que implica también la realización de un poster). En este caso la nota final es una media ponderada de la nota dada por el tutor $(40 \%)$ y la nota del tribunal $(60 \%)$.

\section{Evaluación continua del TFG y función de tutorización}

Seguidamente, pasamos a describir con más detalle cómo está planificada la evaluación continua del TFG (de acuerdo con el esquema mostrado en la imagen anterior) ${ }^{5}$. La presentación del curso se produce el día de la primera sesión grupal (están previstas tres a lo largo del semestre). Obviamente el trabajo del tutor habrá comenzado mucho antes preparando todo el proceso de tutorización y el calendario concreto de sesiones, y esa presentación en particular, pero para el estudiante esta sesión será el tiro de salida. En esta sesión el tutor tiene que explicar a los estudiantes el tipo de trabajo que se espera que sea su TFG; es decir, los requisitos tanto de forma como de contenido que este debe cumplir. Básicamente, comentará brevemente la normativa y las instrucciones ${ }^{6}$ para el TFG e insistirá en que ellos mismos la consulten para informarse de todos los detalles.

\footnotetext{
${ }^{5}$ Orientacions de les Tutories de Treball de Final de Grau. Facultat d'Economia i Empresa. Curs 2015-2016.

${ }^{6}$ Instruccions per a l'Elaboració del Treball de Final de Grau. Facultat d'Economia i Empresa. Curs 2015-16.
} 
Realizado lo anterior, en esta primera sesión el tutor debe también ser capaz de explicar brevemente las diferentes fases por las que el proceso de realización del TFG debería transitar: definición del temaobjetivos-hipótesis, consulta bibliográfica, diseño de la investigación, recogida de datos, análisis de los datos, extracción de conclusiones, redacción de la memoria y, en su caso, presentación del TFG (realización de un poster). Probablemente insistirá más en las primeras cuestiones (tema-objetivos, bibliografía, diseño) que son las que el estudiante tendrá que afrontar antes, obviamente. Proporcionará a los estudiantes, también, una bibliografía básica para que ellos mismos puedan profundizar en el tema de la producción de trabajos académicos de investigación. Por último, probablemente finalizará la sesión realizando alguna indagación sobre el perfil y los intereses y las preferencias de los alumnos que va a "tutorizar" y también con algunas palabras de ánimo.

Tras esa primera sesión se espera que el estudiante produzca y entregue en 3 semanas ( 4 como mucho) un primer documento de trabajo que tiene por nombre "Propuesta de Proyecto de Estudio" (PPE). En esta PPE el estudiante tiene esbozar el trabajo que ha decidido llevar a cabo: tema, objetivos, título, bibliografía, metodología, datos, técnicas de análisis (estadístico) y un calendario previsto. Después de estudiar la propuesta de trabajo presentada por el estudiante, el tutor tiene que convocarlo a una primera sesión individual (están previstas 3 a lo largo del curso) para darle su opinión al respecto y, sobre todo, para corregir las deficiencias que pueda apreciar.

Nuestra propuesta es que el tutor del TFG presente el recurso, el libro de Moodle, en esa primera sesión grupal de presentación. Debería sugerir una primera lectura rápida (necesariamente superficial) del manual por parte del estudiante (en una semana quizás) y una segunda lectura a conciencia de los primeros 4 temas (otra semana). El objetivo es proporcionarle, de forma rápida y concisa, los conocimientos básicos para que sea capaz de generar con éxito la primera evidencia de progreso que es la PPE.

El trámite siguiente a la PPE es la entrega del índice del TFG, lo que supone un paso más en la concreción del trabajo de investigación. También está prevista una nueva sesión grupal. En esta segunda sesión (y en la última; a diferencia de la primera) los estudiantes y no el tutor serán los protagonistas. En la misma, cada uno de ellos ha de explicar al resto el trabajo realizado hasta el momento: la propuesta de investigación y todas las dificultades superadas para llegar a definirla. La puesta en común de las experiencias de cada uno de ellos se supone también generadora de aprendizaje y se pueden aportar sugerencias interesantes para los diferentes trabajos que todavía se encuentran en una fase embrionaria.

Pasados dos meses se espera ya que el estudiante disponga de un borrador del trabajo (como decíamos, el tiempo es muy justo y el trabajo del estudiante debe de ser realmente intensivo ${ }^{7}$ ). Esto implica, obviamente, haber realizado ya el grueso de la investigación: haber recogido (o generado) los datos, haberlos analizado estadísticamente (informáticamente) y haber extraído las conclusiones que den respuesta a los objetivos y las hipótesis que se habían planteado. Los capítulos del libro del 5 al 9 (datos, muestreo, encuestas, técnicas estadísticas, software) están pensados para asistir al estudiante en esta segunda fase y son, por tanto, aquellos que el estudiante debería trabajar antes de abordar esta segunda etapa del trabajo de investigación. Están específicamente relacionados con el análisis estadístico de datos ya que el libro de Moodle está destinado especialmente a estudiantes que quieren aplicar estas técnicas en su TFG.

A continuación, está prevista una segunda sesión individual en la que el tutor, tras leer ese primer borrador de la investigación, le dé al estudiante su opinión y le sugiera correcciones y mejoras. También está planificada una tercera sesión grupal en la que los estudiantes tienen que exponer ya ante sus compañeros los resultados de la investigación. Esta tercera sesión puede entenderse también como un ensayo de la presentación pública del trabajo que algunos estudiantes pueden acabar también realizando.

\footnotetext{
${ }^{7}$ Debemos comentar que la mayoría de estudiantes no matriculan más de una o dos asignaturas además de la del TFG en el semestre en que lo realizan (son estudiantes que están a punto de finalizar sus estudios). Esto permite que la mayoría puedan dedicarse intensamente a la realización de este trabajo.
} 
La última fase es la de la redacción de la memoria del trabajo y, en su caso, la de la presentación pública por medio de un poster y una breve exposición oral. En el libro se dedica el último capítulo a estas cuestiones: desde sugerencias para organizarse el tiempo para escribir, pasando por los contenidos que se deben incluir (y aquellos que no) en cada apartado del documento, hasta ideas para la elaboración de un poster lo más atractivo posible y para una exposición también lo más amena posible. Como en las dos fases anteriores, el estudiante debería leer este último capítulo antes de iniciar la tercera y última etapa del trabajo que es la de la redacción de la memoria.

\section{Descripción del recurso creado: el libro y los cuestionarios}

Como ya se ha indicado, el recurso consiste en un libro de Moodle accesible, por tanto, vía el campus virtual de la universidad. Lo complementan una serie de cuestionarios que, como la herramienta principal, están pensados para que sean gestionados por el tutor de TFG: administrados a los estudiantes cuando convenga e interpretados/evaluados. A continuación, pasamos a describir con detalle estos elementos ${ }^{8}$.

\section{Recurso docente: "libro"}

El libro presenta, lógicamente, la estructura que es de esperar de un producto que lleve tal nombre: es un material didáctico que se divide en capítulos y apartados y, como muestra la imagen inferior, el usuario de la herramienta (el estudiante) dispone en todo momento del índice en un panel a la derecha de la pantalla. Este aspecto hace posible una navegación rápida entre las diferentes páginas, véase Figura 2.

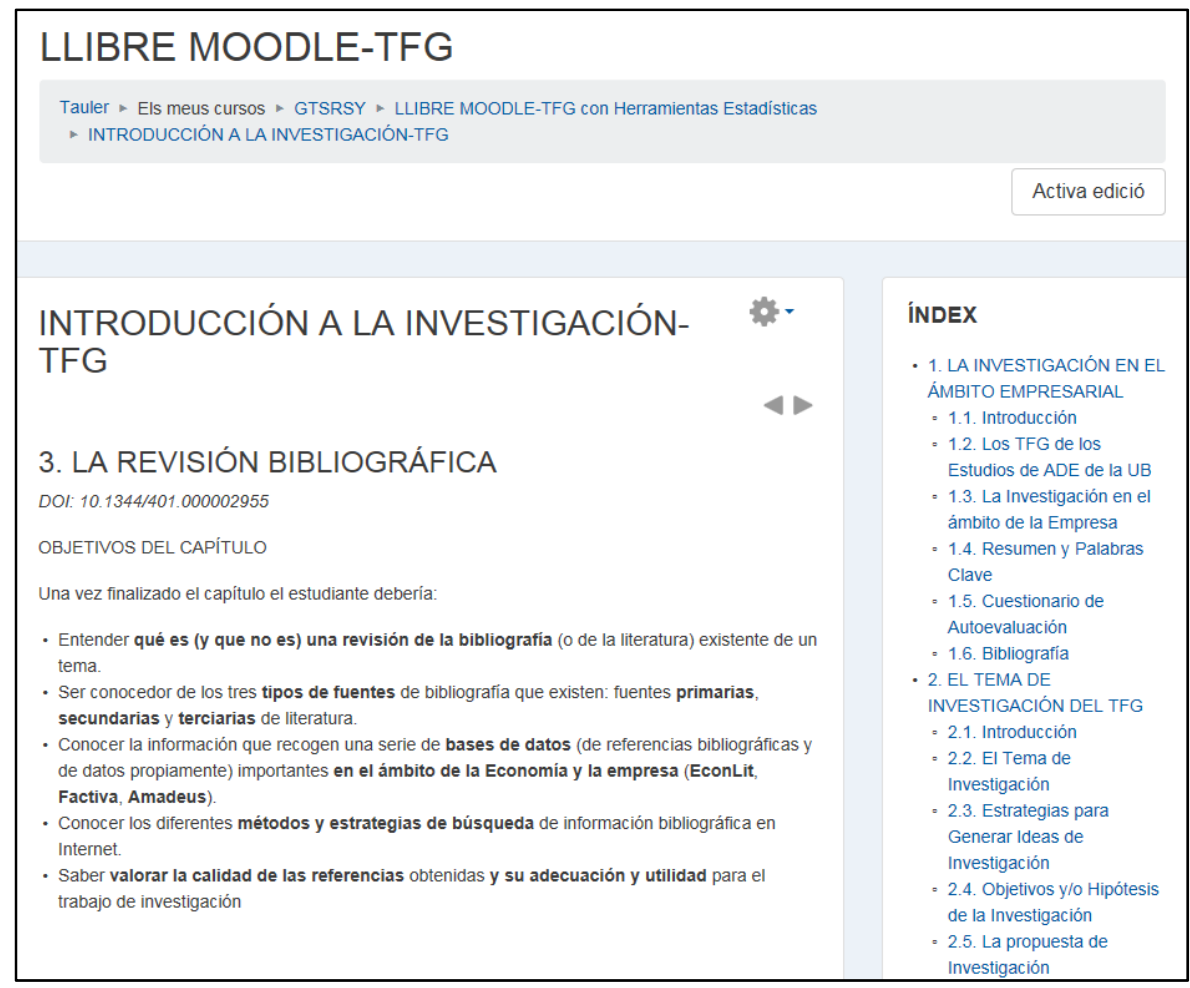

Figura 2: Libro Moodle-TFG

\footnotetext{
${ }^{8}$ El libro de Moodle y los cuestionarios que lo acompañan están localizados en el web del campus virtual de la Universitat de Barcelona. El enlace https://campusvirtual2.ub.edu/course/view.php?id=35450 permite acceder a ellos previa solicitud de inscripción al curso y la aceptación de la misma.
} 
A la hora de decidir y distribuir los contenidos, cada apartado se hace corresponder con una página-web. El objetivo es mostrar las ideas básicas de cada tema o subtema, de forma clara, esquemática o gráfica en muchas ocasiones, para facilitar una consulta rápida por parte de los estudiantes (véase Figura 3). El libro cuenta con un total de 10 capítulos (en el Anexo 1 se muestra el índice) y unas 100 páginas (web) aproximadamente.

El libro comienza con un primer capítulo que, por un lado, expone los detalles y requisitos que la universidad exige al TFG que ha de elaborar el alumno y que, por otro, trata de las particularidades de la investigación en el ámbito de la Gestión Empresarial. Entre estas peculiaridades destaca el énfasis en la aplicabilidad práctica de los resultados de la investigación. A continuación, en una serie de tres capítulos, se tratan las cuestiones de la definición del tema del trabajo, con sus objetivos e hipótesis a contrastar, la revisión de la bibliografía existente sobre el mismo y la decisión del diseño concreto de la investigación.

Sin duda, esta fase inicial de definición (del tema) y de diseño (de la investigación) es una fase crucial; probablemente la más importante. Decisiones erróneas en estas cuestiones serán difíciles de corregir a posteriori y pondrán en riesgo la viabilidad de la investigación y, por tanto, del trabajo. Como se ha comentado en el apartado anterior, los cuatro primeros capítulos del libro deberían leerse a conciencia en las primeras 2-3 semanas para permitir al estudiante elaborar la PPE y el índice del TFG.

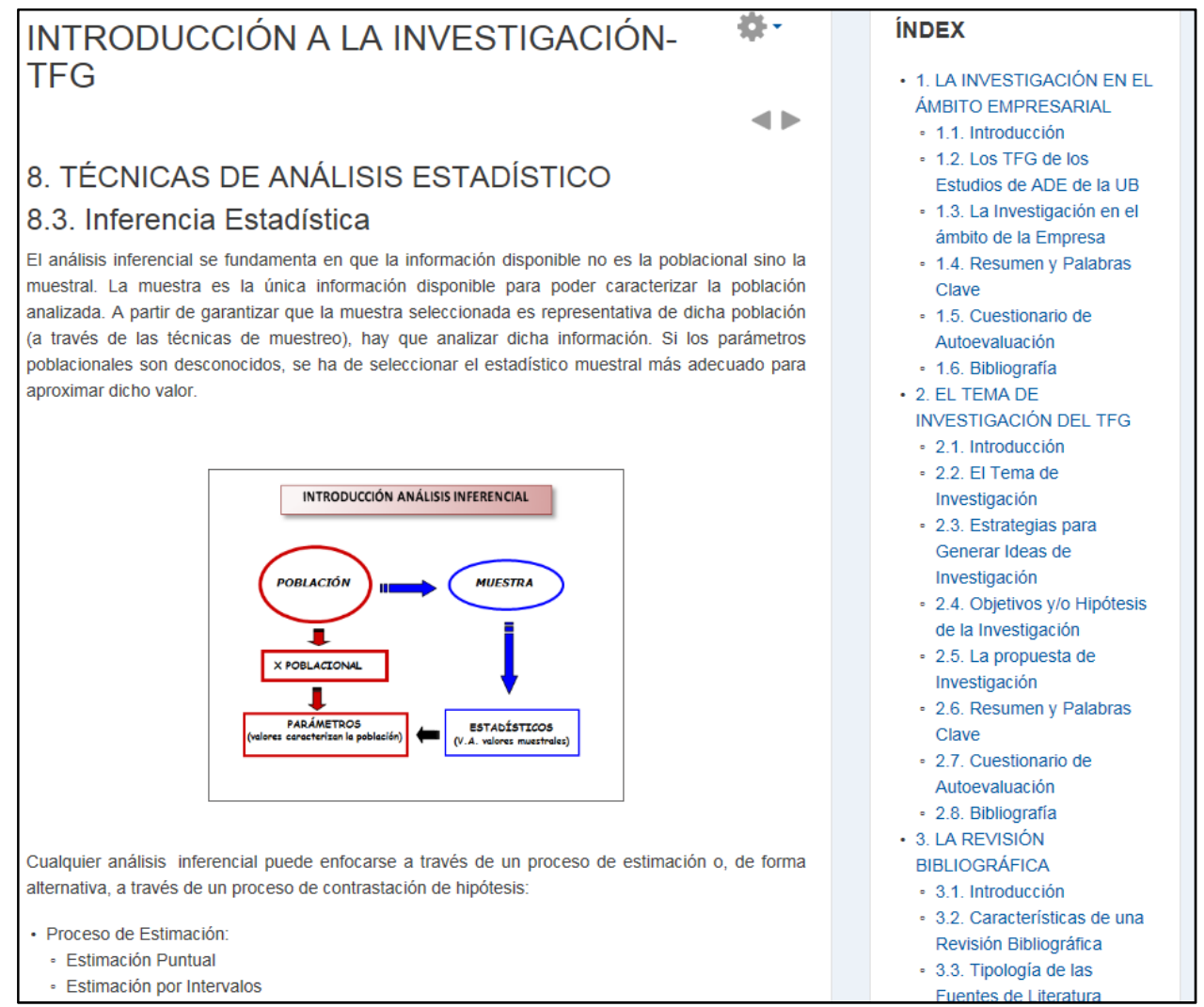

Figura 3: Capítulo de libro

A continuación, hay un segundo bloque de 5 capítulos (del 5 al 9) relacionados todos ellos con el análisis de datos. Los tres primeros hacen referencia a esos datos (fuentes de datos ya existentes y muestreo y encuestas para el caso en que los datos se hayan de producir), el siguiente es una panorámica de técnicas estadísticas disponibles (un recordatorio de lo visto en las diferentes asignaturas de Estadística del Grado) y el último, una panorámica de las herramientas informáticas disponibles. 
Cierra el libro un último capítulo sobre la redacción del trabajo y la presentación pública. Tras la realización de la investigación, esta y sus resultados se tienen que explicar al público. Esto se conseguirá a través de una memoria y quizás también por medio de un poster y una exposición pública. Lógicamente, se recomienda la lectura de este capítulo antes de poner negro sobre blanco los resultados del trabajo de investigación en forma de memoria del TFG.

Todos los capítulos comparten una misma estructura: una introducción, una serie (más o menos larga; depende del tema) de apartados y tres apartados finales de resumen y palabras clave, preguntas de autoevaluación y bibliografía. Creemos que los resúmenes y los breves cuestionarios al final de cada tema pueden ser de gran utilidad para el estudiante; sobre la bibliografía somos, quizás, más escépticos; por el ajustado tiempo que el estudiante tiene. Los cuestionarios de autoevaluación son un elemento que aporta un punto de actividad al material y al aprendizaje y también han de permitir al estudiante y al tutor que los monitoriza detectar aquellas cuestiones de mayor dificultad.

\section{$\underline{\text { Los cuestionarios }}$}

Como se ha dicho anteriormente, junto al libro se diseñan dos cuestionarios para posibilitar el análisis del impacto del material para el alumnado. Hay que señalar que la aprobación por parte de la universidad de un proyecto de investigación docente exige el diseño de este tipo de herramientas de evaluación posterior?.

Un primer cuestionario pretende obtener una aproximación a los conocimientos previos de que dispone el estudiante (ante el reto de elaborar su TFG). Consiste en una treintena de preguntas agrupadas en bloques que hacen referencia a las diferentes fases del proceso a seguir para realizar un trabajo de investigación. Así, hay preguntas relacionadas con la tarea de definir el tema de investigación, otras sobre fuentes bibliográficas, otras sobre datos y otras sobre técnicas de análisis, por citar algunos tipos.

Las preguntas son abiertas y la evaluación de cada pregunta será categórica: Respuesta correcta/regular/incorrecta. El cuestionario previo concluye con dos preguntas diferenciadas de las anteriores: la primera pide al estudiante que haga memoria de los trabajos realizados a lo largo del grado (se pide que haga una descripción breve para poder evaluar la entidad de los mismos) y la segunda le invita a expresar las sensaciones (ansiedad, interés, etc.) que el hecho de tener que hacer el TFG le genera. Finalmente el profesor ha de poder clasificar al estudiante, en función de las diferentes respuestas, como perfectamente preparado (para realizar el TFG), convenientemente preparado, mínimamente preparado o escasamente o no preparado.

Con el objetivo de evaluar el impacto del uso del recurso en los conocimientos del estudiante, este cuestionario inicial de 30 preguntas debe realizarse de nuevo, por parte del estudiante, al finalizar el curso (en este caso, el período de tutorías pensadas para orientar la realización del TFG). Debería observarse una mejora significativa en los conocimientos de la mayoría de los estudiantes. Como ya se ha indicado, el proyecto finalizó el curso anterior y este curso 2017-18 es el primero en que el material estará disponible para los estudiantes. Habrá que esperar, por tanto, unos meses para tener los primeros resultados.

Además de esa evaluación objetiva de la utilidad de la herramienta, se diseña también un segundo cuestionario, más breve, para conocer la valoración subjetiva por parte del estudiante ${ }^{10}$. Para obtener un feedback útil que permita la mejora del material se le pide al estudiante que evalué, además de la utilidad global del libro, la utilidad del mismo en las diferentes fases del proceso. Las preguntas exigen una evaluación numérica por parte del estudiante excepto la última (¿Podrías destacar, por último, los aspectos más positivos y aquellos que, según tu opinión, se podrían mejorar del curso de Moodle que has seguido?) que es abierta.

\footnotetext{
${ }^{9}$ Las preguntas concretas que incluyen estos cuestionarios se pueden consultar en el Anexo 3.

${ }^{10}$ Este segundo cuestionario se muestra también en un anexo (Anexo 3).
} 


\section{Puntos fuertes y limitaciones (futuras mejoras) del material}

Como ya se ha dicho, este curso académico 2017-18 será el primero en que el recurso (el libro de Moodle) es dado a conocer a estudiantes de ADE que van a hacer su TFG bajo la temática particular de Técnicas Estadísticas en la Investigación Empresarial. Por tanto, todavía no es posible presentar algún resultado sobre su impacto en los conocimientos y la utilidad de la herramienta percibida por ellos. Por este mismo motivo también se hace difícil hablar de aciertos y fallos, o limitaciones, del recurso. Pero vamos a intentar hacer alguna reflexión al respecto.

Para empezar, podemos mencionar los criterios principales que tiene la Universidad de Barcelona a la hora de evaluar una propuesta de innovación docente. Recordemos que este proyecto recibió la aprobación por parte del Programa de Millora i Innovació Docent (PMID) de la UB bajo el Vicerrectorado de Política Docente (Marín et al., 2017).

El primer criterio indicado es que el proyecto se plantee como una intervención directa sobre el alumnado con la intención de producir una mejora en los aprendizajes. El segundo, que suponga un cambio o mejora respecto a una situación inicial. Se aprecia que ambos criterios van en la misma línea de que el proyecto tenga un impacto claro, y no ambiguo o indirecto, o lejano, en el aprendizaje de los estudiantes. Creemos que la herramienta que hemos creado cumple claramente este requisito cubriendo una laguna importante en la formación de los estudiantes a los que va destinada; estudiantes que, como ya se ha comentado, tienen unos conocimientos prácticamente nulos (por no haber sido instruidos al respecto, específicamente, a lo largo de sus estudios) acerca del proceso a seguir y la metodología a utilizar para realizar un trabajo de investigación de la envergadura que tiene (o debería tener) el TFG.

El recurso permite al estudiante, con la ayuda de su tutor, seguir a lo largo de la evaluación continuada del TFG una especie de curso online gratuito de metodología en investigación empresarial adaptado a los escasos tres meses de que dispone. Antes este estudiante sólo podía contar con las indicaciones y, quizás, la bibliografía proporcionada por el tutor en las sesiones de tutorización y (no queremos dejar de mencionarlo) el soporte de unas interesantes sesiones que organiza la biblioteca de la facultad sobre algunos aspectos concretos del proceso; sesiones que no constituyen, sin embargo, un curso bien estructurado ni planificado en el tiempo como pretende serlo nuestro material.

Un tercer criterio es que la innovación se desarrolle siguiendo una planificación previa. Como se ha explicado, se ha planificado el uso del material a lo largo del semestre de forma paralela al calendario que la universidad (la facultad) establece para la presentación de las diferentes evidencias de progreso en la elaboración del TFG. Por otro lado, se ha previsto también una difusión gradual, a lo largo de sucesivos cursos académicos, del recurso entre los estudiantes para observar el grado de aceptación y, en su caso, poder realizar algún cambio (mejora) en la herramienta.

Un cuarto criterio es que el proyecto contemple unos indicadores de evaluación de la mejora de los aprendizajes y un quinto que permita disponer de evidencias resultado de la innovación. Como se ha descrito, el proyecto incluye junto al libro una serie de cuestionarios para poder medir de forma objetiva el impacto del recurso en los conocimientos de los estudiantes y de forma más subjetiva el nivel de utilidad que ellos mismos le otorgan. Igualmente, las notas finales de los TFG de los estudiantes que hayan seguido el curso no dejaran de ser una evidencia más del impacto que haya podido tener el material.

El proyecto también se ajusta a diferentes líneas de innovación docente planteadas por el PMID de la UB como aquella que sostiene que la innovación se establece en el marco del entorno virtual de Moodle y supone un desarrollo de las llamadas nuevas formas de aprender; en este caso una forma de aprender a distancia (fuera del entorno de unas clases), en línea (vía Internet; en cualquier momento) y de forma autónoma por parte del estudiante (con el soporte puntual del tutor de TFG). Otra línea hace referencia a la elaboración y difusión de contenidos en abierto. A este respecto, no descartamos una difusión más 
amplia del recurso una vez consideremos que su idoneidad e utilidad han sido convenientemente contrastadas.

Otras líneas hablan de proyectos de innovación que permitan aprendizajes que profesionalicen. Se mencionan, concretamente, proyectos que implique el desarrollo de competencias transversales (genéricas y comunes para todas las titulaciones) y proyectos relacionados con la realización del TFG. Es evidente que la realización del TFG va a requerir movilizar gran parte de los conocimientos y las competencias (habilidades) de la titulación.

Por lo que refiere a la evaluación, el PMID menciona entre otras la autoevaluación, la evaluación continuada y la evaluación entre pares que la herramienta diseñada puede permitir desarrollar. En este caso, la autoevaluación por parte del estudiante es posible gracias a los cuestionarios creados para cada tema del libro y, sobre todo, por la constatación de que consigue (o no) ir superando sin problemas las sucesivas etapas de elaboración del TFG, lo que remite también al concepto de evaluación continuada. La evaluación entre pares también puede ser posible (en las sesiones de tutoría grupales) en la medida en que todos los estudiantes habrán utilizado también el recurso y tendrán unos conocimientos básicos que deberían permitir la crítica constructiva del trabajo realizado por los compañeros.

Para acabar, sobre las metodologías activas de aprendizaje destaca el aprendizaje autónomo, aquel entre iguales, aquel para la elaboración de proyectos y aquel relacionado con la tutorización, entre otros. Creemos que nuestro proyecto permite el desarrollo de estas metodologías especiales de aprendizaje. El aprendizaje autónomo es claro y obligado para el estudiante dado las características de la asignatura del TFG que no tiene docencia (clases presenciales). Al respecto del aprendizaje entre pares, nos remitimos a lo dicho en el apartado anterior sobre las sesiones grupales. Por otro lado, el TFG es claramente un proyecto: un proyecto de investigación que tiene que ser desarrollado en un periodo de unos tres meses al final de los estudios. Y, por último, la herramienta creada ha de permitir una acción tutorial eficiente y ajustada a las necesidades del alumno y a los requerimientos formativos, que son las indicaciones que al respecto hace la universidad.

Comentaremos también algunas limitaciones del material, que en el futuro nos gustaría poder resolver. Probablemente la limitación más importante sea el hecho de que el libro no es un hipertexto, esto es, no es posible una navegación a través de él por conceptos aunque sí sea fácil moverse de un apartado a otro, gracias a que el índice siempre se muestra a la derecha de la pantalla. Efectivamente, una de las ventajas de los textos en soporte digital frente a aquellos en papel es permitir saltar al instante (navegar) de unos contenidos a otros. Es una limitación de la herramienta Libro de Moodle que no lo permite.

También nos habría gustado contemplar diferentes itinerarios de lectura en función de las necesidades específicas de los estudiantes o del deseo del tutor. De hecho, en un primer momento se pensó en hacer uso de la herramienta Lección de Moodle pero finalmente se descartó la idea por su complejidad notablemente superior a la de la herramienta Libro. Quizás, ahora que existe el libro, sería interesante ofrecer también los contenidos en otro formato distinto y más flexible, de las lecciones.

En esa línea, nos gustaría también integrar más el recurso creado en el que sería un campus virtual de la asignatura TFG. Por ejemplo, los cuestionarios de autoevaluación podrían ser cuestionaros de Moodle para facilitar el análisis de los resultados. También el libro podría incorporar sugerencias para temas de discusión o debate entre los estudiantes del grupo de TFG; debates que podrían realizarse gracias a la herramienta Foro de Moodle.

En general creemos que el recurso tal y como ahora está ya puede ser de una utilidad importante para los estudiantes (y los tutores) y, en este sentido, creemos que los objetivos del proyecto se cumplieron, pero también que es posible enriquecer o potenciar esa utilidad y el aprendizaje de los alumnos vinculando el libro a otras herramientas de la plataforma Moodle. 
Por último, si la herramienta se demuestra útil nos podríamos plantear replicarla para algún otro tipo de TFG de los contemplados en la normativa.

En la Tabla 1 se resume los puntos comentados en este apartado.

PUNTOS FUERTES DEL RECURSO (LIBRO DE MOODLE)

- El proyecto supone una intervención directa sobre el alumnado con la intención de producir una mejora en los aprendizajes. Supone un cambio o mejora respecto a una situación inicial.

- La innovación se desarrolla siguiendo una planificación.

- El proyecto contempla unos indicadores de evaluación de la mejora de los aprendizajes y permite disponer de evidencias resultado de la innovación.

- El proyecto se ajusta a diferentes líneas de innovación contempladas en el PMID: innovación en el marco del entorno virtual de Moodle, elaboración y difusión de contenidos en abierto y proyectos que permiten aprendizajes que profesionalicen.

- El proyecto implica nuevas metodologías de aprendizaje: aprendizaje autónomo, aprendizaje ligado a una actividad de tutorización, aprendizaje asociado a la realización de proyectos y aprendizaje entre pares.

- El proyecto implica diferentes tipos de evaluación: evaluación continuada, autoevaluación y evaluación por pares.

\section{PUNTOS DÉBILES Y POSIBLES MEJORAS}

- El libro no es un hipertexto por una limitación tecnológica; porque la herramienta utilizada (la herramienta Libro de Moodle) no lo permite.

- Podría ser interesante ofrecer los contenidos del actual libro en un formato distinto y más innovador utilizando la herramienta Lección también de la plataforma Moodle.

- Está pendiente incrementar el grado de integración del recurso, del libro, en el campus de la asignatura del TFG con el objeto de sacar más partido a la plataforma (Moodle).

- Existiría la posibilidad de replicar la herramienta para dar soporte a la realización de otros tipos de TFG previstos en la normativa.

Tabla 1: Puntos fuertes y débiles

\section{Conclusiones}

Como profesores-tutores de estudiantes del Grado de ADE-UB a punto de finalizar sus estudios, que se ven en la necesidad de hacer un trabajo de fin de grado para completarlos, venimos detectando ciertas carencias o limitaciones en las competencias de estos alumnos de cara a la realización de ese trabajo de investigación. En especial los estudiantes parecen tener especial dificultad para afrontar con éxito la que sería la primera fase del proceso: la de la definición del tema de investigación (objetivos y preguntas) y la de su diseño (datos y técnicas).

Creemos que esta situación, que sería interesante estudiar en profundidad, exhaustivamente, se debe a que no existe en el Plan de Estudios una asignatura dedicada a la materia de la investigación en el ámbito de las ciencias sociales y, en concreto, en el ámbito de la Empresa; a que muchos estudiantes consultan muy poca bibliografía a lo largo de sus estudios, especialmente trabajos de investigación (monografías, artículos en revistas académicas); y a que muchos apenas han realizado (en el grado) trabajos de una cierta envergadura.

En este contexto recae sobre el tutor de TFG la responsabilidad de versar, en el breve lapso de tiempo de unas pocas semanas, y mediante una serie de (también pocas) sesiones grupales e individuales, a los estudiantes en la materia. Es cierto que tanto desde la Coordinación de la asignatura de TFG de la facultad como de la Biblioteca hay aportaciones de recursos interesantes (documentación, sesiones) sobre temas 
específicos, pero no un material completo, integrado y con las características del que hemos elaborado y comentamos aquí.

El recurso, el libro, puede ser utilizado por el tutor como material (manual/curso) básico que recomienda al estudiante al inicio del semestre para que este lo consulte de forma paralela (con cierta antelación) a la realización de su investigación. Su tarea sería, por tanto, la de calendarizar la consulta por temas del libro y la de comprobar que las diferentes cuestiones han sido comprendidas bien por el estudiante y que efectivamente se aplican a la hora de avanzar en la realización del TFG.

Este recurso docente presenta ciertas características innovadoras (se presentó, de hecho, como propuesta de innovación docente a la universidad y la solicitud fue aprobada) relacionadas básicamente con el hecho de ofrecerse en soporte digital sobre la plataforma virtual Moodle a través del campus virtual de la universidad.

El proyecto finalizó recientemente (el curso pasado) y todavía no es posible evaluar el impacto del recurso en el aprendizaje de los alumnos ni conocer la valoración que hacen de la utilidad de la herramienta. Se han diseñado una serie de cuestionarios con esta finalidad. También está pendiente acabar de integrar bien la herramienta (el libro) en el campus virtual de la asignatura de TFG con las otras de que dispone Moodle.

\section{Referencias}

Comisió de TFG de la Facultad d'Economia i Empresa (2015). Normativa de Treball de Final de Grau de la Facultat d'Economia i Empresa de la Universitat de Barcelona. Aprobada por la Comissió Académica de la Facultat en Julio del 2012. Modificada por la misma Comisión el 15 de Octubre de 2015.

Facultat d'Economia i Empresa (2016). Instruccions per a l'elaboració del Treball de Final de Grau. Curs 2016-17.

Facultat d'Economia i Empresa (2016). Orientacions de les Tutories de Treball de Final de Grau. Curs 2016-17.

Marín, S., et al. (2017). Introducción a la Investigación - TFG. http://hdl.handle.net/2445/110067. Dipòsit Digital UB. (DOI: 10.1344/401.000002955).

Bibliografia utilizada en la elaboración del recurso (libro de Moodle) presentado en el artículo

Bhattacherjee, A. (2012) Social science research principles, methods, and practices (2nd ed.). Tampa: Scholar Commons, University of South Florida.

Bryman, A., y Bell, E. (2011) Business research methods (3rd ed.). Oxford: Oxford University Press.

Collis, J., y Hussey, R. (2009) Business research: a practical guide for undergraduate \& postgraduate students (3rd ed). Basingstoke [etc.]: Palgrave Macmillan.

Cooper, D.R., y Schindler, P.S. (2008) Business research methods (10th ed). Boston: McGraw-Hill Irwin.

Ghauri, P.N., y Grønhaug, K. (2005) Research methods in business studies: a practical guide (3rd ed.). Harlow [etc.]: Financial Times Prentice Hall.

Gill, J., Johnson, P., y Clark, M. (2010) Research methods for managers (4th ed.). London: SAGE.

Hair, J.F. (2011) Essentials of business research methods (2nd ed.). Armonk, N.Y.: M.E. Sharpe, Inc.

Saunders, M.N.K., Lewis, P., y Thornhill, A. (2012) Research methods for business students (6th ed.). Harlow [etc.]: Pearson.

Wilson, J. (2010) Essentials of business research: a guide to doing your research project. London: SAGE. 


\section{Anexo 1. Índice detallado del libro del Moodle}

\section{LA INVESTIGACIÓN EN EL ÁMBITO EMPRESARIAL}

1.1 Introducción

1.2 Los TFG de los Estudios de ADE de la UB

1.3 La Investigación en el ámbito de la Empresa

1.4 Resumen y Palabras Clave

1.5 Cuestionario de Autoevaluación

1.6 Bibliografía

\section{EL TEMA DE INVESTIGACIÓN DEL TFG}

2.1 Introducción

2.2 El Tema de Investigación

2.3 Estrategias para Generar Ideas de Investigación

2.4 Objetivos y/o Hipótesis de la Investigación

2.5 La propuesta de Investigación

2.6 Resumen y Palabras Clave

2.7 Cuestionario de Autoevaluación

2.8 Bibliografía

\section{LA REVISIÓN BIBLIOGRÁFICA}

3.1 Introducción

3.2 Características de una Revisión Bibliográfica

3.3 Tipología de las Fuentes de Literatura Científica

3.4 Fuentes Secundarias de Literatura

3.5 Fuentes terciarias de Literatura

3.6 Bases de Datos en el ámbito de la Economía y la Empresa

3.7 Búsquedas en Internet

3.8 Planificación de la Búsqueda

3.9 Evaluación y Gestión de las Referencias

3.10 Resumen y Palabras Clave

3.11 Cuestionario de Autoevaluación

3.12 Bibliografía

\section{ASPECTOS METODOLÓGICOS Y DE DISEÑO}

4.1 Introducción

4.2 Filosofías en el Campo de la Investigación Científica

4.3 Aproximaciones: Deductiva e Inductiva

4.4 El diseño de la Investigación. Estudios Exploratorios, Descriptivos y Causales. Experimentos, Estudios observacionales y Análisis de Casos

4.5 Métodos: Cuantitativos y Cualitativos. Estudios de Corte Transversal y de Serie Temporal

4.6 Fiabilidad y Validez de la Investigación Científica

4.7 Cuestiones de Tipo Ético

4.8 Resumen y Palabras Clave

4.9 Cuestionario de Autoevaluación

4.10 Bibliografía

\section{FUENTES DE DATOS}

5.1 Introducción

5.2 Datos Abiertos: filosofía

5.3 Fuentes Oficiales de Datos

5.4 Algunos Ejemplos con Coste 
5.5 Resumen y Palabras Clave

5.6 Cuestionario de Autoevaluación

5.7 Bibliografía

6 MUESTREO

6.1 Introducción

6.2 Población. Muestra

6.3 Técnicas de Muestreo Probabilísticos

6.4 Técnicas de Muestreo No Probabilísticos

6.5 Elección del Tamaño Muestral

6.6 Resumen y Palabras Clave

6.7 Cuestionario de Autoevaluación

6.8 Bibliografía

\section{LA ENCUESTA}

7.1 Introducción

7.2 Etapas: Diseño de una Encuesta

7.3 Diseño del cuestionario

7.4 Tipologías de Cuestionarios

7.5 Reglas Básicas: Formulación de Preguntas

7.6 Ordenación de Preguntas

7.7 Validación: Prueba Piloto

7.8 Administración: Medios

7.9 Trabajo de Campo

7.10 Resumen y Palabras Claves

7.11 Cuestionario de Autoevaluación

7.12 Bibliografía

8 TÉCNICAS DE ANÁLISIS ESTADÍSTICO

8.1 Introducción

8.2 Técnicas de Análisis Descriptivo

8.3 Inferencia Estadística

8.4 Modelo de Regresión

8.5 Modelo de Series Temporales

8.6 Otras Técnicas: Técnicas Multivariantes

8.7 Resumen y Palabras Clave

8.8 Cuestionario de Autoevaluación

8.9 Bibliografía

9 SOFTWARE PARA EL ANÁLISIS DE DATOS

9.1 Introducción

9.2 Tipología de Software para Tratamiento de Datos

9.3 Software Comercial

9.4 Software libre

9.5 Resumen y Palabras Clave

9.6 Cuestionario de Autoevaluación

10 REDACCIÓN Y PRESENTACIÓN DE LA MEMORIA DEL TFG

10.1 Introducción

10.2 Cuándo, Cómo y Dónde Escribir

10.3 Estructura de Apartados de la Memoria del Trabajo de Investigación

10.4 Contenidos de la Memoria.1

10.5 Contenidos de la Memoria.2 
10.6 Realización de un Póster sobre el TFG

10.7 Presentación Oral del TFG

10.8 Resumen y Palabras Clave

10.9 Cuestionario de Autoevaluación

10.10 Bibliografía

11 SOLUCIONES CUESTIONARIOS

\section{Anexo 2. Capturas de pantalla del libro de Moodle}

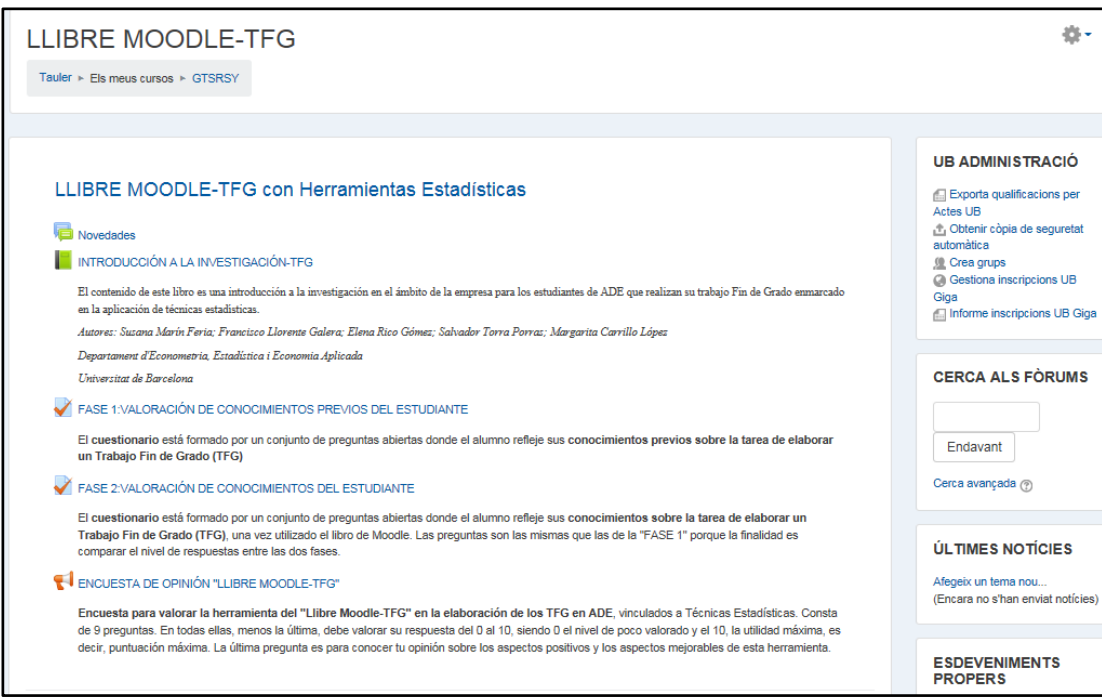

Figura 4: Pantalla de presentación del material (campus virtual del libro) a los estudiantes

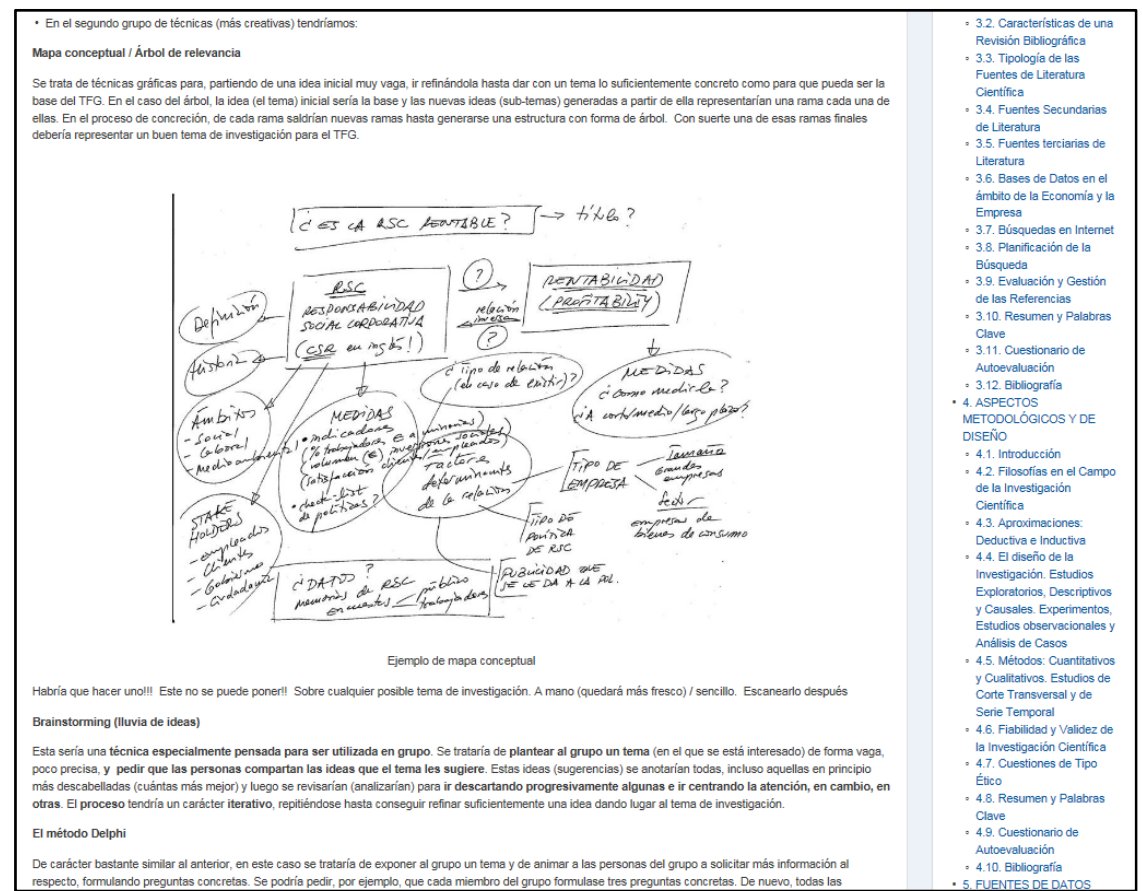

Figura 5: Página de muestra del libro 


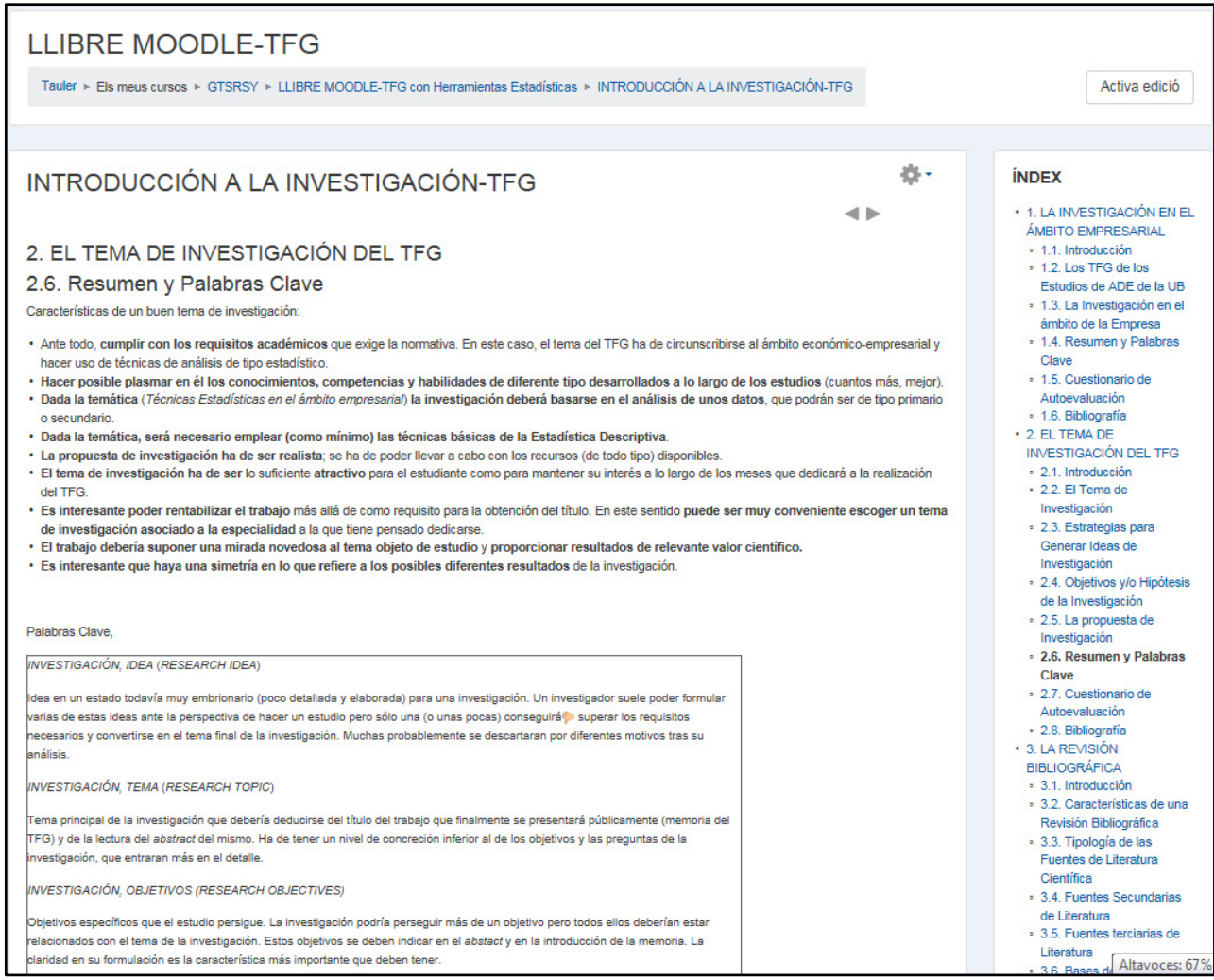

Figura 6: Página de resumen y palabras claves de un tema

\begin{tabular}{|c|c|}
\hline \multicolumn{2}{|l|}{ LLIBRE MOODLE-TFG } \\
\hline Tauler - Els meus cursos - GTSRSY = LIBRE MOODLE-TFG con Herramientas Estadisticas - INTRODUCCIÓN A LA INVESTIGACIÓN-TFG & Activa edició \\
\hline 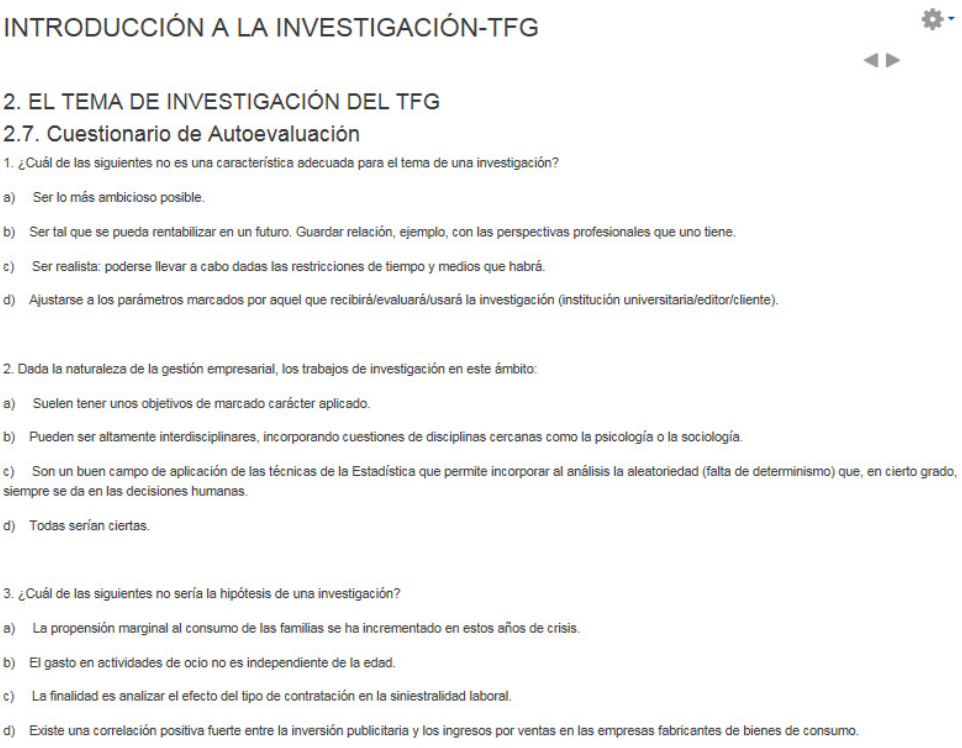 & 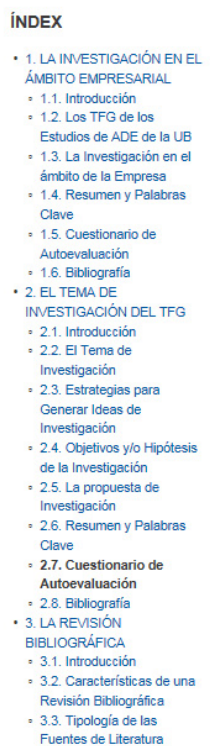 \\
\hline
\end{tabular}

Figura 7: Página de un cuestionario de autoevaluación de un tema 


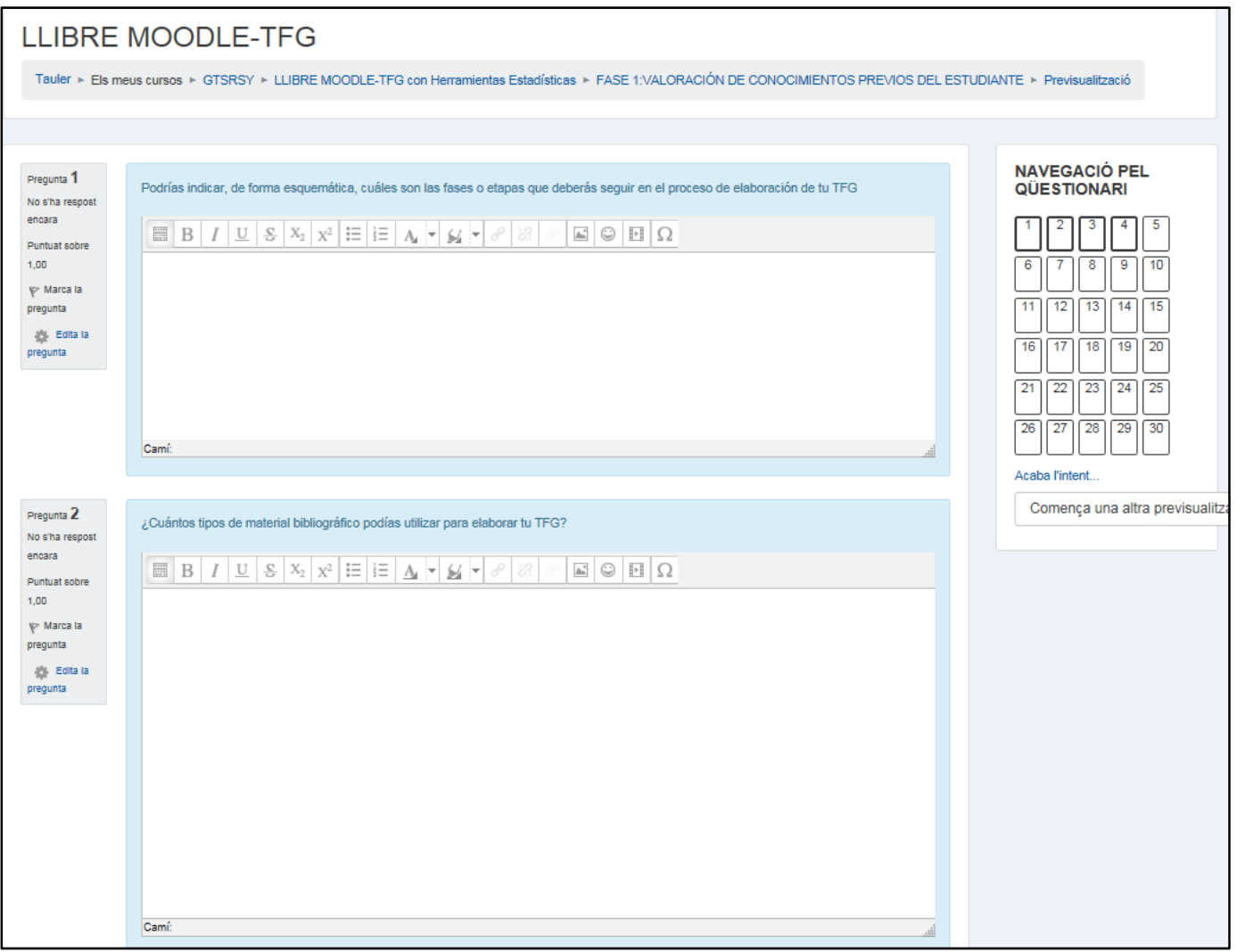

Figura 8: Cuestionario de conocimientos previos (una de las páginas)

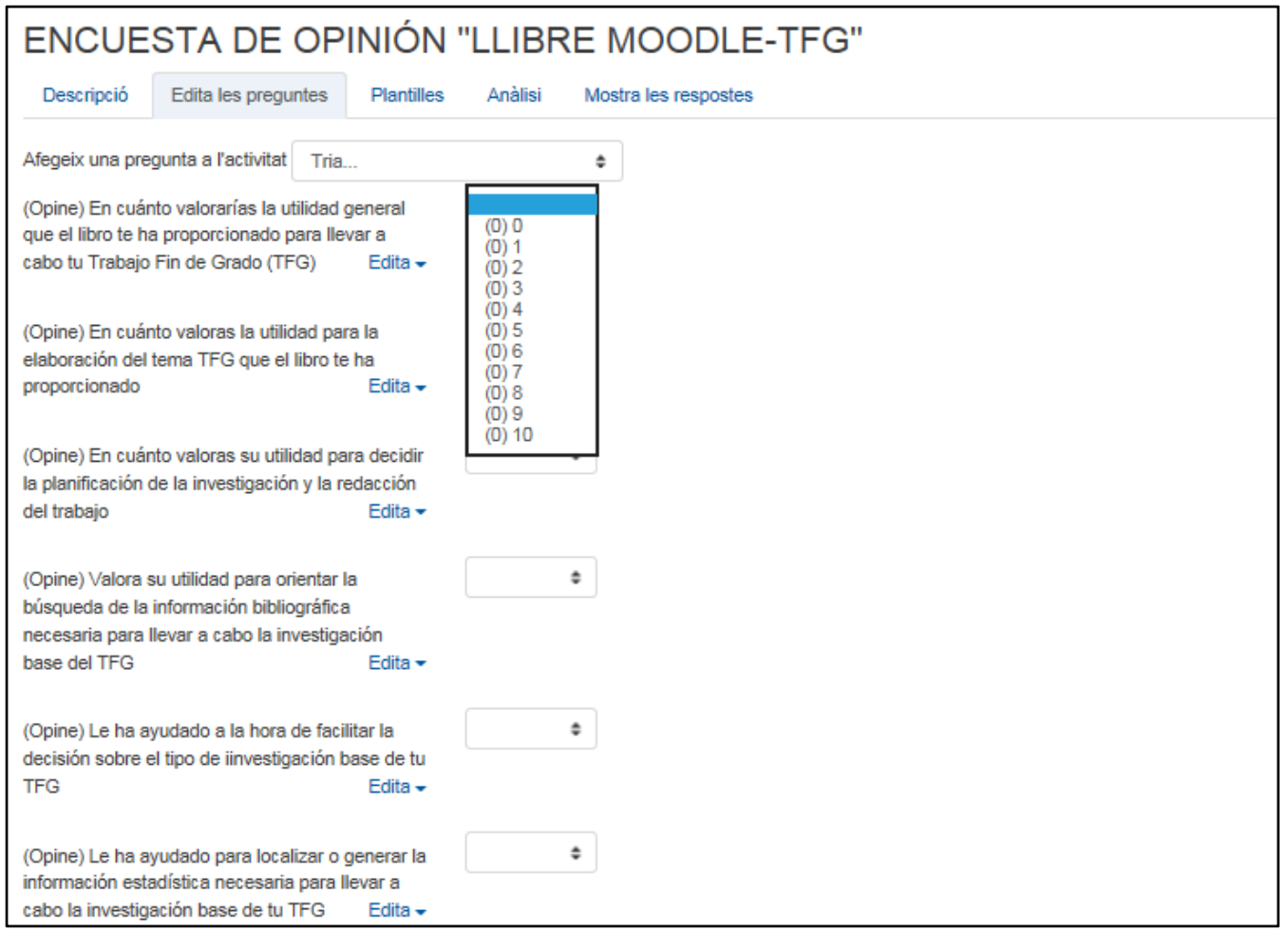

Figura 9: Cuestionario de evaluación del material 


\section{Anexo 3. Cuestionarios de I) Conocimientos previos y II) Valoración del recurso}

\section{CUESTIONARIO DE VALORACIÓN DE CONOCIMIENTOS PREVIOS DE CARA A LA REALIZACIÓN DEL TFG}

(Estudiantes de ADE que han escogido la temática de TFG en Técnicas Estadísticas en un Entorno Empresarial).

El cuestionario está formado por un conjunto de preguntas abiertas y la valoración de su resultado se hará en función de la proximidad de las respuestas proporcionadas por el estudiante a las consideradas correctas.

1. ¿Podrías indicar, de forma esquemática, cuáles son las fases o etapas que deberías seguir en el proceso de elaboración de tu TFG?

Bibliografía

2. ¿Qué tipos diferentes de material bibliográfico podrías utilizar para elaborar tu TFG?

3. ¿Cómo evaluarías la calidad de una publicación académica?

4. ¿Y la calidad de un artículo en este tipo de publicaciones?

5. ¿Cuál es el problema principal de la información disponible en Internet?

Tema de Investigación

6. ¿Qué requisitos básicos crees que ha de tener una propuesta de trabajo de investigación como la que ha de servir de base a tu TFG?

7. ¿Cuál es la diferencia entre una investigación pura y una aplicada?

8. ¿Cuál ha de ser la característica principal de una hipótesis de investigación?

9. ¿Qué quiere decir que una propuesta de investigación es simétrica en los resultados? Diseño de la investigación- Recogida de datos

10. Explica brevemente la diferencia entre un proceso deductivo y uno inductivo, en el marco de la investigación científica

11. ¿Cuál es la característica principal de un estudio longitudinal?

12. En un "survey" (investigación muestral), ¿cuáles son las cuestiones a decidir, previas a su realización?

13. ¿Qué factores determinan el tamaño de la muestra (en un estudio muestral)?

14. La encuesta es un método de recogida de información importante en las Ciencias Sociales. Detalla algunos sesgos y malas prácticas que pueden ocurrir cuando se utiliza este instrumento de recogida de datos

15. ¿Cómo definirías el sesgo de no-respuesta en el marco de un estudio basado en una encuesta?

16. ¿Qué es un cuestionario auto administrado?

17. Explica qué se quiere decir cuando se habla de una fuente de información estadística secundaria. Análisis de los datos y términos estadísticos

18. ¿Cuáles son las características de un método de muestreo aleatorio?

19. ¿Podrías nombrar tres tipos diferentes de muestreo aleatorio?

20. ¿En qué consiste un muestreo por cuotas?

21. ¿Qué es el error muestral?

22. ¿Qué es el error estándar?

23. ¿Podrías mencionar un par de técnicas de análisis estadístico que permitan estudiar la relación entre dos variables?

24. ¿Qué es una serie temporal?

25. ¿Qué quiere decir que unos datos son de corte transversal?

26. ¿Podrías mencionar tres técnicas de Análisis Multivariante? Redacción del documento del TFG

27. ¿Qué estructura básica ha de tener el documento de presentación de un trabajo de investigación?

28. ¿Qué suele incluirse, además de las conclusiones, en el apartado de Conclusiones de un trabajo de investigación?

29. ¿Qué es un estilo de citación? 
30. ¿Qué información se ha de incluir al citar una página-web que se ha consultado para realizar un trabajo de investigación?

Preguntas finales:

¿Cuántos trabajos de cierta entidad has realizado a lo largo de tus estudios de ADE hasta el momento? (Indícalos y descríbelos brevemente)

¿Cómo definirías (con qué calificativos) la situación emocional que te genera el tener que elaborar tu TFG?

CUESTIONARIO DE VALORACIÓN (POR PARTE DEL ESTUDIANTE) DEL LIBRO DE MOODLE DE SOPORTE A LA ELABORACIÓN DE LOS TFG-ADE EN TÉCNICAS ESTADÍSTICAS

1. De 0 a 10 , ¿en cuánto valorarías la utilidad general que el curso te ha proporcionado para llevar a cabo tu Trabajo de Fin de Grado (TFG)?

2. De 0 a 10 , ¿en cuánto valorarías la utilidad para la elección del tema del TFG que el curso te ha proporcionado?

3. De 0 a 10 , ¿en cuánto valorarías su utilidad para decidir la planificación de la investigación y la redacción del trabajo?

4. De 0 a 10 , ¿en cuánto valorarías su utilidad para orientar la búsqueda de la información bibliográfica necesaria para llevar a cabo la investigación base del TFG?

5. De 0 a 10 , ¿en cuánto valorarías su utilidad para facilitar la decisión sobre el tipo o diseño de la investigación base de tu TFG?

6. De 0 a 10 , ¿en cuánto valorarías su utilidad para facilitar la localización o la generación de la información estadística necesaria para llevar a cabo la investigación base de tu TFG?

7. De 0 a 10 , ¿en cuánto valorarías su utilidad para facilitar la elección de las técnicas estadísticas más adecuadas a aplicar a los datos base de la investigación de tu TFG?

8. De 0 a 10 , ¿en cuánto valorarías su utilidad para facilitar las decisiones sobre la estructura del documento final del TFG, su redacción (estilo, etc.), la elaboración de la bibliografía y su presentación?

9. ¿Podrías destacar, por último, los aspectos más positivos y aquellos que, según tu opinión, se podrían mejorar del curso de Moodle que has seguido? 